\title{
Preconditioning methods influence tumor property in an orthotopic bladder urothelial carcinoma rat model
}

\author{
KOZO MIYAZAKI $^{1,2}$, YUJI MORIMOTO ${ }^{2}$, NOBUHIRO NISHIYAMA ${ }^{1}$, HIROYUKI SATOH $^{3}$, \\ MASAMITSU TANAKA ${ }^{2}$, NARIYOSHI SHINOMIYA ${ }^{2}$ and KEIICHI ITO ${ }^{4}$
}

\author{
${ }^{1}$ Division of Clinical Biotechnology, Center for Disease Biology and Integrative Medicine, Graduate School of Medicine, \\ The University of Tokyo, Tokyo 113-0033; ${ }^{2}$ Department of Integrative Physiology and Bio-Nano Medicine, \\ National Defense Medical College, Saitama 359-8513; ${ }^{3}$ Department of Urology, Tokyo Metropolitan \\ Children's Medical Center, Tokyo 183-8561; ${ }^{4}$ Department of Urology, National \\ Defense Medical College, Saitama 359-8513, Japan
}

Received May 14,2013; Accepted September 11, 2013

DOI: $10.3892 / \mathrm{mco} .2013 .214$

\begin{abstract}
Urothelial carcinoma (UC) is an extremely common type of cancer that occurs in the bladder. It has a particularly high rate of recurrence. Therefore, preclinical studies using animal models are essential to determine effective forms of treatment. In the present study, in order to establish an orthotopic bladder UC animal model with clinical relevance, the effects of preconditioning methods on properties of the developed tumor were evaluated. The bladder cavity was pretreated with phosphate-buffered saline (PBS), acid-base, trypsin (TRY) or poly (L-lysine) (PLL) and then rat UC cells (AY-27) $\left(4 \times 10^{6}\right.$ cells) were inoculated. The results demonstrated that, two weeks later, the tumorigenic rate $(88 \%)$ and tumor count (2.3 per rat) were not significantly different among the preconditioning methods, whereas tumor volume and invasion depth into bladder tissue were significantly different. Average tumor volumes were $>50 \mathrm{~mm}^{3}$ in the PBS and acid-base-treated groups and $<10 \mathrm{~mm}^{3}$ in the TRY- and PLL-treated groups. The percentage of invasive tumors (T2 or more advanced stage) was $\sim 75 \%$ of total tumors in the PBS- and acid-base-treated groups, whereas the percentages were reduced in the TRY- and PLL-treated groups (58 and 32\%, respectively). Non-invasive tumors (Ta or T1) accounted for $54 \%$ of tumors in the PLL-treated group, which was 2-5-fold higher than the percentages in the remaining groups. Properties of the developed tumor in the rat orthotopic UC model were different depending on preconditioning methods. Therefore, different animal models suitable for a discrete preclinical examination
\end{abstract}

Correspondence to: Professor Yuji Morimoto, Department of Integrative Physiology and Bio-Nano Medicine, National Defense Medical College, Namiki 3-2, Tokorozawa, Saitama 359-8513, Japan E-mail: moyan@ndmc.ac.jp

Key words: phosphate-buffered saline, acid-base, trypsin, polylysine, ultrasonography may be established by using the appropriate preconditioning condition.

\section{Introduction}

Approximately $90 \%$ of cancers in the human bladder are urothelial carcinoma (UC) and the recurrence rate is $>50 \%$ (1) when standard therapy such as transurethral resection is conducted. Carcinoma in situ, in particular, has a high recurrence rate and often progresses to invasive carcinoma (2) and patients therefore have to undergo invasive surgical treatment (total cystectomy), resulting in a reduction to the quality of life.

Preclinical studies using experimental animal models are crucial for evaluation of effective treatment for human UC. The experimental animal model should have properties of UC similar to those of human UC, the established tumor should be orthotopic and the aspects of progression and invasion should be similar to those of human UC. To establish such an animal model of orthotopic UC, preconditioning of the bladder mucosa is frequently carried out prior to UC cell inoculation into the bladder cavity. Various preconditioning methods, including chemical treatment (3-7), biological modification (8) and physical injury $(9,10)$, have been conducted previously, with the tumorigenic rate varying from $6-100 \%(3,5,10)$. The rate and aspects of tumor growth may be associated with the manner of applied preconditioning (7). However, the results of previous statistical analyses have not been reported.

In this study, four different preconditioning treatments of orthotopic UC were examined in order to establish an animal model, and the associations between preconditioning and patterns of tumor growth and development were evaluated. The four preconditionings were selected according to the following criteria: establishment of the model was possible in a short period of time by preconditioning, the preconditioning itself was not too invasive for host tissues, and the tumor was capable of being colonized widely in the bladder cavity by preconditioning. The developed tumors were examined to determine tumorigenic incidence and the effect on invasion depth of the tumor in each preconditioning group. 
Table I. Tumorigenic rate (no. of rats with tumors) and invasion depth of the tumor in each preconditioning group.

\begin{tabular}{lcccccccccc}
\hline \multirow{2}{*}{$\begin{array}{l}\text { Preconditioning } \\
\text { method }\end{array}$} & $\begin{array}{c}\text { No. of } \\
\text { rats }\end{array}$ & T0 & Tis & Ta & T1 & T2a & T2b & T3a & T3b & $\begin{array}{c}\text { No. of rats } \\
\text { with tumors (\%) }\end{array}$ \\
\cline { 3 - 9 } PBS & 35 & 4 & 2 & 0 & 6 & 7 & $\underline{11}$ & 4 & 1 & $31(89)$ \\
Acid base & 33 & 4 & 4 & 0 & 3 & 8 & $\underline{11}$ & 3 & 0 & $29(88)$ \\
TRY & 34 & 1 & 6 & 1 & 7 & $\underline{13}$ & 5 & 1 & 0 & $33(97)$ \\
PLL & 35 & 7 & 4 & 2 & $\underline{13}$ & 8 & 1 & 0 & 0 & $28(80)$ \\
Total & 137 & 16 & 16 & 3 & 29 & 36 & 28 & 8 & 1 & $121(88)$ \\
\hline
\end{tabular}

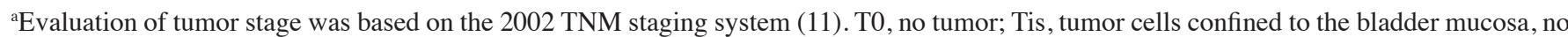
papillary tumor; Ta, papillary tumor; T1, tumor invading bladder submucosa, but not beyond; T2a, tumor invading one-half of the muscle layer, but not through it; T2b, tumor invading beyond one-half of the muscle layer, but not through the muscle layer; T3a, tumor penetrating through the bladder wall, no apparent invasion of adjacent organs; T3b, tumor penetrating through the bladder wall, apparent invasion of adjacent organs. If a rat had more than two categories (Tis, Ta, T1, T2a, T2b, T3a and T3b), it was allocated to the highest category. Underlined numbers are the maximum numbers in each group. PBS, phosphate-buffered saline; TRY, trypsin; PLL, poly (L-lysine).

\section{Materials and methods}

Cell culture. Cell line AY-27 was established as a primary UC in Fischer F344 rats by feeding them $\mathrm{N}$-[4-(5-nitro-2-furyl)-2-thiazolyl] formamide. The cells were grown in RPMI-1640 (Invitrogen, Carlsbad, CA, USA) with $10 \%$ heat-inactivated fetal bovine serum (SAFC Biosciences, Lenexa, KS, USA) and $40 \mu \mathrm{g} / \mathrm{ml}$ of gentamicin (Invitrogen) at $37^{\circ} \mathrm{C}$ under a $5 \% \mathrm{CO}_{2}$ atmosphere in an incubator. The cells were passaged and passages 5-15 were used in subsequent experiments. The cells were washed with Dulbecco's phosphate-buffered saline (DPBS), harvested with $0.25 \%$ trypsin/ EDTA (Invitrogen), and then centrifuged at $200 \mathrm{x}$ g for $5 \mathrm{~min}$ and resuspended in a culture medium $\left(1 \times 10^{7}\right.$ cells $\left./ \mathrm{ml}\right)$.

Animal model of bladder tumor and preconditioning methods. All animal experiments were performed according to the protocol approved by the Ethics Committee for Laboratory Animals of the National Defense Medical College.

Female Fischer F344 rats at 5,7 or 11 weeks old (Charles River Laboratories, Yokohama, Japan) were each anesthetized with $30 \mathrm{mg} / \mathrm{kg}$ of intraperitoneal pentobarbital (Dainippon Sumitomo Pharma, Osaka, Japan). Lidocaine hydrochloride jelly $2 \%$ (AstraZeneca, Osaka, Japan) was applied to the external urethral orifice and an 18-gauge plastic intravenous cannula (Terumo, Tokyo, Japan) was introduced into the bladder cavity using a $0.64 \mathrm{~mm}$ guide wire (RF-GA25053, Terumo). Four different preconditioning methods were performed: i) PBS preconditioning group $(n=35)$, which involved washing the bladder cavity twice with a $0.8 \mathrm{ml}$ of DPBS using a $1-\mathrm{ml}$ syringe via the cannula, and injecting $0.4 \mathrm{ml}$ of the cell suspension in RPMI- $1640\left(1 \times 10^{7}\right.$ cells $\left./ \mathrm{ml}\right)$ into the bladder, then maintaining for $1 \mathrm{~h}$. ii) Acid-base preconditioning group $(\mathrm{n}=33)$, which involved injection of $0.4 \mathrm{ml}$ of $0.1 \mathrm{M}$ hydrochloric acid into the bladder and stirring using a back-and-forth motion of a syringe via the cannula for $15 \mathrm{sec}$ (1 stroke/sec). Then, $0.4 \mathrm{ml}$ of $0.1 \mathrm{M}$ potassium hydroxide $(\mathrm{KOH})$ was injected for neutralization and agitated using another syringe for $15 \mathrm{sec}(1 \mathrm{stroke} / \mathrm{sec})$. The neutralized liquid was removed, and the cell suspension was injected and left for $1 \mathrm{~h}$. iii) Trypsin (TRY) preconditioning group ( $\mathrm{n}=34)$, which involved washing the bladder cavity once with $0.8 \mathrm{ml}$ of DPBS. Then, $0.5 \mathrm{ml}$ of $0.25 \%$ trypsin solution (Invitrogen) was injected into the bladder using another syringe. The solution was agitated using a back-and-forth motion for $25 \mathrm{sec}(1 \mathrm{stroke} / \mathrm{sec})$ and left for $15 \mathrm{~min}$. The trypsin solution was agitated for a further $25 \mathrm{sec}$ and removed, and then the cell suspension was injected into the bladder and left for 1 h. iv) Poly (L-lysine) (PLL) preconditioning group ( $\mathrm{n}=35$ ), which involved washing the bladder cavity once with $0.8 \mathrm{ml}$ of DPBS. Then, $0.5 \mathrm{ml}$ of $0.11 \%$ PLL solution (Sigma-Aldrich) was injected into the bladder using another syringe and the solution was agitated using a back-and-forth motion for $25 \mathrm{sec}(1 \mathrm{stroke} / \mathrm{sec})$ and left for $15 \mathrm{~min}$. The PLL solution was agitated again for $25 \mathrm{sec}$ and removed, and then the cell suspension was injected into the bladder and left for $1 \mathrm{~h}$.

In situ ultrasound imaging of the bladder tumor. Two weeks after the inoculation of UC cells, under anesthesia (intraperitoneal pentobarbital, $30 \mathrm{mg} / \mathrm{kg}$ ), the developed bladder tumor was non-invasively observed using an ultrasound imaging system with a high-resolution ultrasound probe (RMV704 and Vevo 770, VisualSonics Inc., Toronto, Canada). Prior to the examination, we confirmed preliminarily that the detectable minimum size of the tumor was $1 \mathrm{~mm}$ in this system.

Tumor volume. Each rat was sacrificed two weeks after UC cell inoculation and the bladder was extracted. The anterior wall of the bladder was vertically incised from the ureterovisical junction to the bladder apex. Visually recognized elevated lesions $>1 \mathrm{~mm}$ in diameter were considered tumors, and then the number of tumors in the bladder was counted and the volume of each tumor was measured. The tumor volume (V) was calculated using the formula: $\mathrm{V}\left(\mathrm{mm}^{3}\right)=(\mathrm{a} \times \mathrm{b} \times \mathrm{c}) \pi / 6$, where a, major axis; b, minor axis; and c, height.

Histopathological examination. The extracted bladder was fixed with $4 \%$ paraformaldehyde buffer and was processed 
A
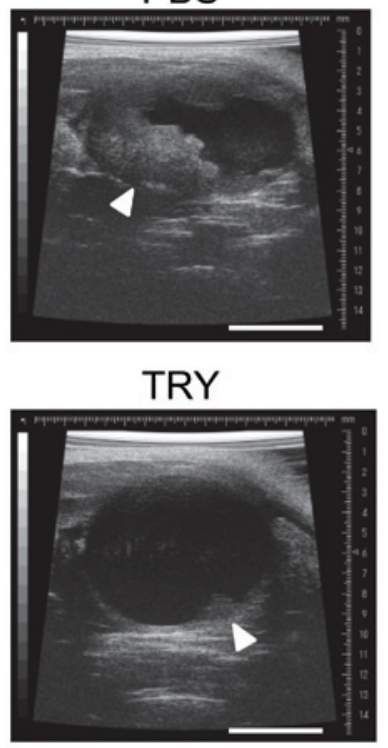

B

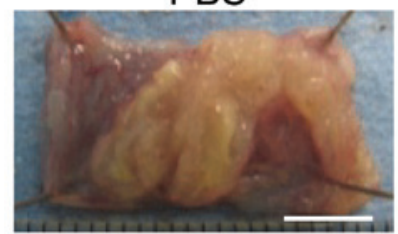

TRY

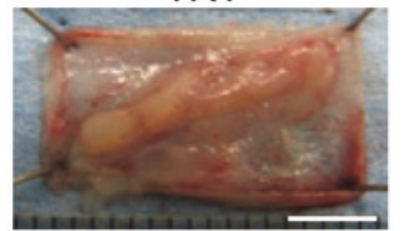

Acid-Base

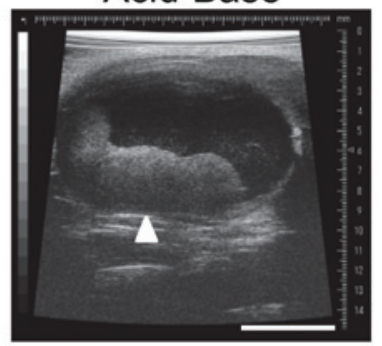

PLL

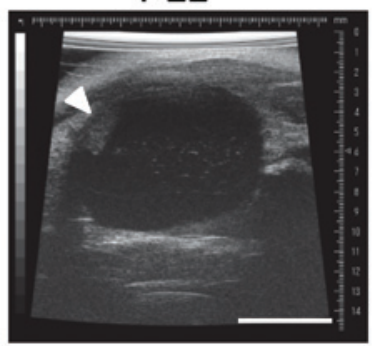

Acid-Base

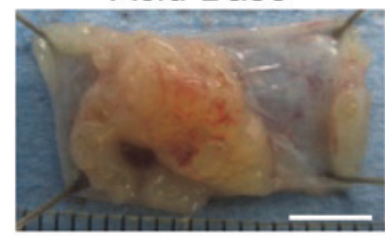

PLL

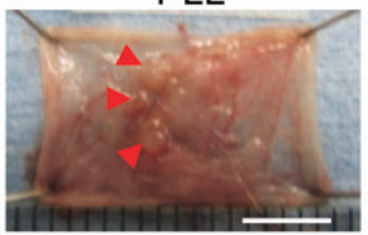

Figure 1. (A) Axial views of ultrasound imaging of the rat urinary bladder and (B) macroscopic images of the bladder tumor. Two weeks following tumor cell inoculation, the ultrasound imaging was conducted and bladders were extracted. The bladders were longitudinally incised at the anterior wall and extended: the upper and lower panels of each image correspond to the apex and base of the bladder, respectively. (A) In ultrasound images, upper panel, ventral direction; lower panel, dorsal direction; left side, leftward; right side, rightward. White arrow shows tumor lesion. Red arrow shows microtumors. Bar, $5 \mathrm{~mm}$.

for H\&E staining. The invasion depth of atypical cells in the bladder wall was evaluated microscopically. Tumor stage (11) was scored for the highest category per rat (Table I).

Statistical analysis. Data are expressed as the means \pm SD. The one-way analysis of variance test followed by Tukey's post-hoc test were used to obtain data pertaining to the tumor volume and tumor count. Pearson's Chi-square test was used to obtain data of invasion depth and tumorigenic rate. $\mathrm{P}<0.05$ was considered to indicate a statistically significant difference.

\section{Results}

Tumorigenic incidence. The tumorigenic incidence ranged from $80-97 \%$ (average $88 \%$ ), and there was no significant difference among the preconditioning groups $(\mathrm{P}=0.18)$ (Table I).

A
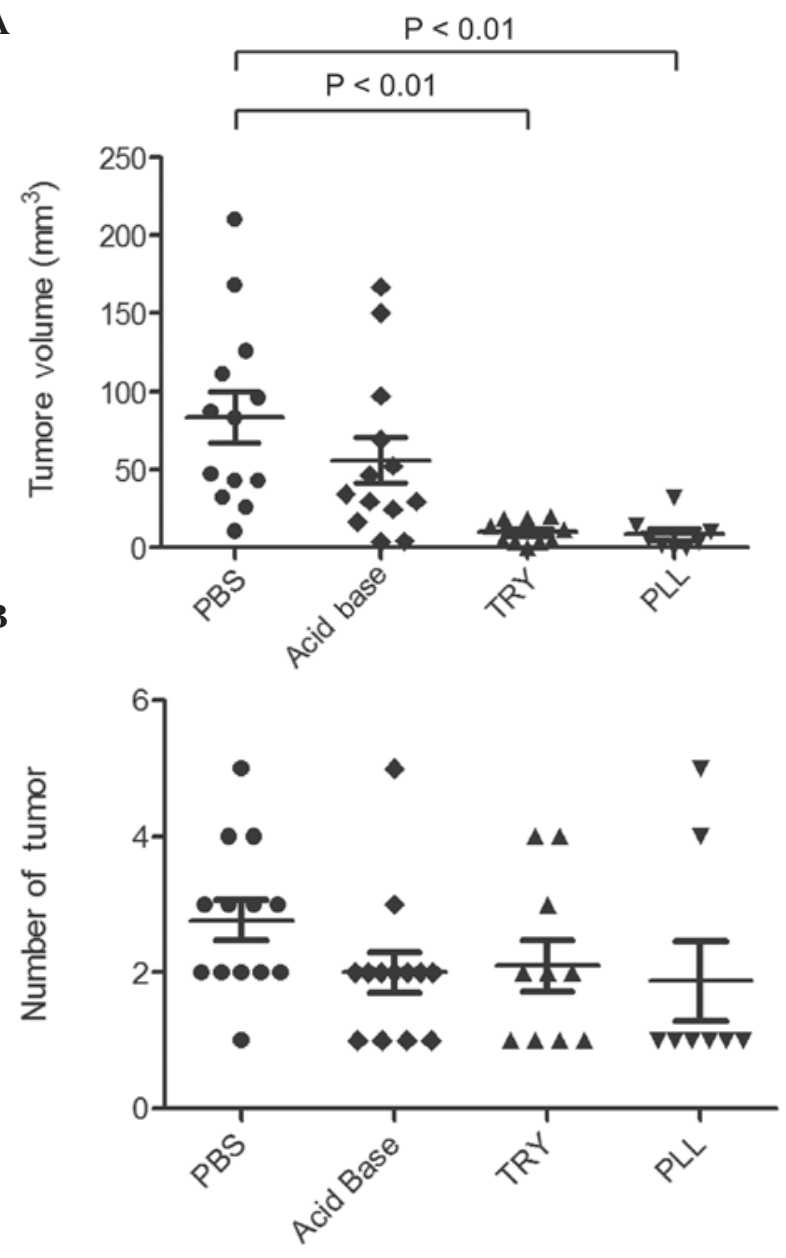

Figure 2. (A) Tumor volume and (B) number of tumors in each preconditioning group. A mass of $>1 \mathrm{~mm}$ in the long axis that protruded on the mucosa was identified as a tumor. The tumor volume and number of tumors per bladder were determined macroscopically. A mass of $<1 \mathrm{~mm}$ in the long axis was not evaluated. Individual data and the means \pm SD are shown in each graph. $\mathrm{n}=13,13,10$ and 8 for phosphate-buffered saline (PBS), acid-base, trypsin (TRY) and poly (L-lysine) (PLL), respectively.

The tumorigenic rate showed a tendency to decrease with the age of the rat, with the number of rats with tumors/number of inoculated rats being 9/9,31/35 and $6 / 9$ at 6,8 and 12 weeks old, respectively. However, no significant difference among the age groups was observed $(\mathrm{P}=0.10)$.

Volume of developed tumors and their number in the bladder. The ultrasound imaging system demonstrated that there was a high frequency of large tumors of $>10 \mathrm{~mm}$ in long axis in the PBS and acid-base groups, while there was a high frequency of small tumors with maximum size $<3-4 \mathrm{~mm}$ in long axis in the TRY and PLL groups (Fig. 1A). Macroscopically, the maximum long axis of the tumor was frequently $10 \mathrm{~mm}$ or more in the PBS and acid-base groups, while it was frequently from 1-5 $\mathrm{mm}$ in the TRY and PLL groups (Fig. 1B). The mean values of tumor volume were $83.4 \pm 59.0 \mathrm{~mm}^{3}$ in the PBS group, $55.8 \pm 52.4 \mathrm{~mm}^{3}$ in the acid-base group, $9.9 \pm 7.5 \mathrm{~mm}^{3}$ in the TRY group and $8.6 \pm 10.8 \mathrm{~mm} 3$ in the PLL group. Significant differences were observed between the PBS and TRY or PLL groups (Fig. 2A). The mean value of tumor counts was 2.3 per rat and there was no significant difference among the preconditioning groups (Fig. 2B). 

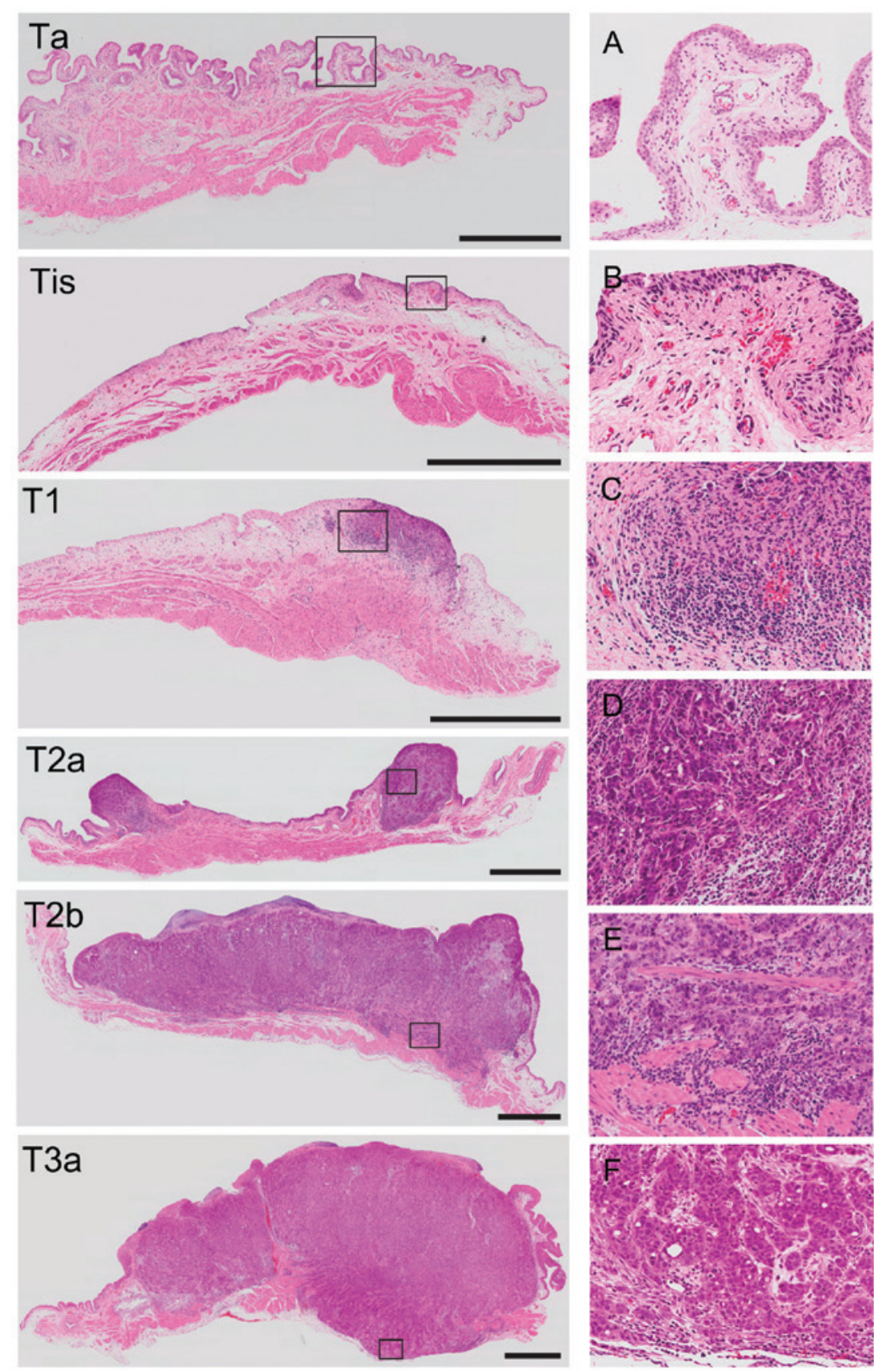

Figure 3. Histopathological images of bladder tumors (H\&E staining) at each tumor stage (left-upper corner of each image). Right-sided images are magnified views of the section inside a square in each left image. Bar, $1 \mathrm{~mm}$. In Ta, papillae are developed and usually short. (A) The nuclear-to-cytoplasmic (N/C) ratio of cells is moderately increased, nuclei have irregularly distributed chromatin, and nucleoli are prominent. In Tis, bladder CIS replaces normal urothelium and partially or entirely covers the bladder lumen surface. (B) A moderate increase in the N/C ratio in cells is evident. In T1, tumor cells invade the submucosa. (C) Low to moderate dysplastic cells (grade I-II) and concentrated inflammatory cells are evident. In the advanced stages, tumor cells invade the bladder muscle (T2) and penetrate the bladder wall (T3). (D-F) Tumor cells show an increase in the N/C ratio with markedly increased variation in nuclear size and shape, and mitotic figures (grade III).

Invasion depth of the tumor. Bladder tissues were histopathologically examined regardless of tumor existence $(n=121)$. $\mathrm{H} \& \mathrm{E}$ staining of the cells revealed a moderately increased nuclear-to-cytoplasmic index, high mitotic activity and marked nuclear pleomorphism; however, no significant pathological difference was observed among the preconditioning groups. Invasion depth of the tumor was from Tis to T3b (Fig. 3) and was significantly different among the groups (PBS vs. PLL, $\mathrm{P}=0.001$; acid-base vs. PLL, $\mathrm{P}=0.001$ ) (Table I). Ratios of invasive tumor type (T2 or T3) to total tumors were 74, 76, 58 and
$32 \%$ in the PBS, acid-base, TRY and PLL groups, respectively, suggesting that the frequency of invasive tumor was higher in the PBS and acid-base groups compared with the PLL group and that the frequency of non-invasive tumor (Tis, Ta or T1) was higher in the PLL group (Table I).

\section{Discussion}

UC is a common type of cancer in the human bladder. Animal models resembling human models are crucial in the 
identification and development of effective treatment strategies for this disease. Four preconditioning methods were established in the present study to establish an orthotopic bladder UC animal model. The results showed no significant difference in tumorigenic incidence or the number of tumors per animal among the preconditioning groups, suggesting that modification or alteration in the substrate (bladder mucosa) resulting from preconditioning has little effect on adhesiveness of the substrate to cells.

By contrast, highly invasive tumors and tumors with a large volume developed in the PBS and acid-base preconditioning groups, while less invasive tumors with a small volume tumor developed in the TRY and PLL preconditioning groups. Considering these results combined with the abovementioned findings (no significant difference in tumorigenic incidence or the number of tumors per animal among the preconditioning groups), tumor size and invasion depth are more likely to be affected by the modified property of cancer cells due to preconditioning than by the modified property of the substrate (bladder mucosa) due to preconditioning.

A plausible reason for the formation of small tumors is inhibited proliferation due to a decrease in the viability of tumor cells exposed to PLL or TRY residue in the bladder mucosa. PLL is known to exhibit cellular toxicity (12). PLL binds tightly to the cell membrane and induces morphological changes (13), leading to membrane defects (14). Trypsin has also been reported to play a tumor-suppressive role in human carcinoma (15). Exposure of cells to trypsin downregulates superficial integrins (16), making cells weak and, occasionally, leading to apoptosis. In the case of TRY, another plausible explanation for the formation of small tumors is the modification of cell-to-cell interaction. Trypsin is involved in cell dissociation, and renders cells as non-adhesive to each other (17). Although AY-27 cells are known to be aggregative $(18,19)$, TRY residue in the bladder mucosa may inhibit cell aggregation.

However, the inhibition of cell viability may not have occurred in the case of preconditioning using PBS or acid-base as the residues resulting from these preconditionings do not exhibit cellular toxicity. Even if a residue existed, chemical or biological action with regard to cellular toxicity and cell-to-cell interaction was unanticipated by PBS, nor was neutralized potassium chloride resulting from acid-base preconditioning.

Results of this study suggest that the ratio of invasive tumor types (T2 or T3) to total tumors was $>70 \%$ in acid-base treatment, while that of invasive tumor types has been reported to be $<35 \%(3,18)$. This discrepancy might be due to differences in the age of rats and/or the inoculated cell numbers. Generally, the developed tumor size is larger in a young host compared to that found in an old host (20), rats at 11 weeks old or older (body weights of 150-200 g) have been used in previous studies $(3,18,21)$, whereas we used 8 -week-old rats $(120 \mathrm{~g})$. Moreover, the tumor becomes larger when numerous cells are inoculated $(7,22)$. In previous studies, $\leq 1.5 \times 10^{6}$ cells were inoculated $(7,22)$, whereas we conducted inoculation of $4 \times 10^{6}$ cells.

At present, there are no reports in which the association between preconditioning and a tumor characteristic such as invasion depth or tumor size is described. To the best of our knowledge, our results demonstrated for the first time that a variety of bladder tumor models may be established by applying different preconditionings. Preconditioning using PBS or acid-base is likely to establish a highly invasive and large volume tumor, while preconditioning using PLL may establish a superficial and small volume tumor.

In conclusion, the association between preconditioning methods and properties of a bladder tumor were evaluated in an orthotopic bladder UC model. Large $\left(>50 \mathrm{~mm}^{3}\right)$ and highly invasive tumors were established in the PBS or acid-base preconditioning group, while small $\left(<10 \mathrm{~mm}^{3}\right)$ and non-invasive tumors were established in the PLL preconditioning group. The difference in preconditioning did not have any significant effect on the tumorigenic rate.

\section{Acknowledgements}

The authors would like to thank Ms. K. Urano and Ms. M. Muraoka for their technical assistance and critical comments. This study was supported by a grant from the New Energy Industrial Technology Development Organization of Japan (Grant No. P06042) to N.N. and a Grant-in-Aid for Scientific Research (Grant No. 23300174) from the Ministry of Education, Culture, Sports, Science and Technology of Japan to Y.M.

\section{References}

1. Sylvester RJ, van der Meijden AP, Oosterlinck W, et al: Predicting recurrence and progression in individual patients with stage Ta T1 bladder cancer using EORTC risk tables: a combined analysis of 2596 patients from seven EORTC trials. Eur Urol 49: 466-475, 2006.

2. Lamm DL: Carcinoma in situ. Urol Clin North Am 19: 499-508, 1992.

3. Xiao Z, McCallum TJ, Brown KM, et al: Characterization of a novel transplantable orthotopic rat bladder transitional cell tumour model. Br J Cancer 81: 638-646, 1999.

4. Zhou JH, Rosser CJ, Tanaka M, et al: Visualizing superficial human bladder cancer cell growth in vivo by green fluorescent protein expression. Cancer Gene Ther 9: 681-686, 2002.

5. Watanabe T, Shinohara N, Sazawa A, et al: An improved intravesical model using human bladder cancer cell lines to optimize gene and other therapies. Cancer Gene Ther 7: 1575-1580, 2000.

6. Arentsen HC, Hendricksen K, Oosterwijk E and Witjes JA: Experimental rat bladder urothelial cell carcinoma models. World J Urol 27: 313-317, 2009.

7. Loskog A, Ninalga C, Hedlund T, Alimohammadi M, Malmstrom PU and Totterman TH: Optimization of the MB49 mouse bladder cancer model for adenoviral gene therapy. Lab Anim 39: 384-393, 2005.

8. Ramesh N, Ge Y, Ennist DL, et al: CG0070, a conditionally replicating granulocyte macrophage colony-stimulating factor - armed oncolytic adenovirus for the treatment of bladder cancer. Clin Cancer Res 12: 305-313, 2006.

9. Shapiro A, Kelley DR, Oakley DM, Catalona WJ and Ratliff TL: Technical factors affecting the reproducibility of intravesical mouse bladder tumor implantation during therapy with Bacillus Calmette-Guerin. Cancer Res 44: 3051-3054, 1984.

10. Bisson JF, Parache RM, Droulle P, Notter D, Vigneron C and Guillemin F: A new method of implanting orthotopic rat bladder tumor for experimental therapies. Int J Cancer 102: 280-285, 2002.

11. TNM Classification of Malignant Tumours, 6th edition (UICC). Hoboken (ed). Wiley-Blackwell, NJ, 2002.

12. Fischer D, Li Y, Ahlemeyer B, Krieglstein J and Kissel T: In vitro cytotoxicity testing of polycations: influence of polymer structure on cell viability and hemolysis. Biomaterials 24: 1121-1131, 2003.

13. Arnold LJ Jr, Dagan A, Gutheil J and Kaplan NO: Antineoplastic activity of poly(L-lysine) with some ascites tumor cells. Proc Natl Acad Sci USA 76: 3246-3250, 1979.

14. Reuter M, Schwieger C, Meister A, Karlsson G and Blume A: Poly-l-lysines and poly-l-arginines induce leakage of negatively charged phospholipid vesicles and translocate through the lipid bilayer upon electrostatic binding to the membrane. Biophys Chem 144: 27-37, 2009. 
15. Yamashita K, Mimori K, Inoue H, Mori M and Sidransky D: A tumor-suppressive role for trypsin in human cancer progression. Cancer Res 63: 6575-6578, 2003.

16. Patel Y, Rahman S, Siddiqua A, Wilkinson JM, Kakkar VV and Authi KS: Functional characterization of PM6/13, a beta3-specific (GPIIIa/CD61) monoclonal antibody that shows preferential inhibition of fibrinogen binding over fibronectin binding to activated human platelets. Thromb Haemost 79: 177-185, 1998.

17. Takeichi M: The cadherins: cell-cell adhesion molecules controlling animal morphogenesis. Development 102: 639-655, 1988.

18. Hendricksen K, Molkenboer-Kuenen J, Oosterwijk E, Hulsbergen-van de Kaa CA and Witjes JA: Evaluation of an orthotopic rat bladder urothelial cell carcinoma model by cystoscopy. BJU Int 101: 889-893, 2008.
19. El Khatib S, Berrahmoune S, Leroux A, Bezdetnaya L, Guillemin F and D'Hallewin MA: A novel orthotopic bladder tumor model with predictable localization of a solitary tumor. Cancer Biol Ther 5: 1327-1331, 2006.

20. Pili R, Guo Y, Chang J, Nakanishi H, Martin GR and Passaniti A: Altered angiogenesis underlying age-dependent changes in tumor growth. J Natl Cancer Inst 86: 1303-1314, 1994.

21. Gronlund-Pakkanen S, Wahlfors J, Talja M, et al: The effect of photodynamic therapy on rat urinary bladder with orthotopic urothelial carcinoma. BJU Int 92: 125-130, 2003.

22. Gunther JH, Jurczok A, Wulf T, et al: Optimizing syngeneic orthotopic murine bladder cancer (MB49). Cancer Res 59: 2834-2837, 1999. 